\title{
Investigation of digestive system disorders with electrogastrogram using wavelet transform denoising
}

\author{
Gopu G. ${ }^{* 1}$, Neelaveni R. ${ }^{1}$, Porkumaran K. ${ }^{2}$, Shekar M.G. ${ }^{3}$ \\ ${ }^{1 *}$ Department of EEE, PSG College of Technology, Coimbatore- 04,Tamilnadu,India, \\ gopugovindasamy@gmail.com, rnv@eee.psgtech.ac.in \\ ${ }^{2}$ Dr. N.G.P. Institute of Technology, Coimbatore, 48, Tamilnadu, India, porkumaran@gmail.com \\ ${ }^{3}$ Department of Urology, SRM Hospital and Research Institute, Kattankulathur, Tamilnadu, India, \\ drmgshekarkumarah@yahoo.in
}

\begin{abstract}
A digestive system disorders are the very important problem in human being universally. As a panacea, a preliminary investigation method is proposed before the endoscopy procedure is called Electrogastrography. Electrogastrogram [EGG] is obtained from the human being cutaneously, which is noninvasive, easy procedure over endoscopy. EGG recorded for a 180 human being includes the normal individual and patients with digestive system disorder such us Nausea, Dyspepsia, Vomiting, ulcer, etc. A database is created for the analysis purpose. During the acquisition of EGG, the analog signal's numeric values are recorded as .bio file format using the data scope. Wavelet transform is used for the analysis of EGG to find the deviation in frequency and Power from the power spectrum estimate plot obtained for disorders patients compare to normal Individual. Wavelet Transform analysis includes the principle component analysis, denosing of the signal and power spectrum estimation. In the proposed wavelet analysis, the data of a patient or normal individual is included for analysis. The EGG signal is reconstructed with a data obtained from the data scope. This signal undergoes principle component analysis to remove the noise in the EGG signal to obtain de-noised signal and it is plotted for power spectral density estimation with Welch power spectral Density Estimation. As a result of the proposed method the power variation is found to be a range of $40-52 \mathrm{~dB}$ and frequency is detected as $0.06 \mathrm{~Hz}$ to $0.075 \mathrm{~Hz}$ for ulcer patient $0.02 \mathrm{~Hz}$ to $0.04 \mathrm{~Hz}$ for dyspepsia patient and $0.05 \mathrm{~Hz}$ to $0.058 \mathrm{~Hz}$ for Normal Individual. The above said finding supports the physician in the diagnosis of digestive system disorders at fair amount of accuracy.
\end{abstract}

Keywords: Electrogastrogram, Nausea, Dyspepsia, Vomiting, ulcer, Wavelet Transform, Welch power spectral Density Estimation

\section{Introduction}

Electrogastrography is a noninvasive method for the recording of gastric myoelectrical activity that controls gastric motility $[1,2]$. Since the first measurement of the EGG was reported 75 years ago by W.C Alvarez. The first recording of gastro electrical activity is performed in dogs by C.F. Code and J.A.Marleh in the year 1974 [3] and in human this procedure is recorded in 1978 [4] by R.L.Telander et al. But the progress in this field has been very slow, especially compared with other cutaneous electrophysiological measurements, such us the Electroencephalography, Electrocardiography etc., because of its difficulty in data acquisition, lack of understanding, etc., Due to the advancement in quantitative analysis of the EGG, more and more physicians and biomedical researchers interested in this field. The abnormality arises due to recurrent nausea, vomiting, Dyspepsia, Stomach ulcer, Cyclic vomiting syndrome, etc which signals that the stomach is not emptying food normally. If the EGG is abnormal, it confirms that the problem probably is with the stomach's muscles or the nerves that control the muscles. This paper deals with the novel approach of recording of the electrical signals that travel through the muscles of the stomach and control the muscle's contraction and it denoised with wavelet transform. The denoised EGG signal's frequency in $\mathrm{Hz}$ and power in $\mathrm{dB} / \mathrm{Hz}$ is obtained with Welch power spectral Density Estimation due to its clear indication of parameters compared to Periodogram power spectral Density Estimation. The EGG can be considered as an experimental procedure since its exact role in the diagnosis of digestive disorders of the stomach has not been defined yet. This paper is organized as follows: Section 1, introduction about the survey and brief explanation about the analysis EGG. Section 2, explains about the EGG. Section 3, includes brief explanation about the anatomy of the stomach. Section 4, illustrate the electrode positioning for recording EGG signals. Section 5, illustrate about the materials and methodology adopted and denoising of the EGG signal using wavelet denoising. Section 6, explains about the acquisition procedure for EGG. Section 7, includes results and discussion. Section 8, includes the summary of this paper and further expansion method. Section 9, includes the acknowledgement to the experts. Section 10, includes the reference papers for this research paper.

\section{Electrogastrogram}

An EGG is similar to an electrocardiogram of the heart. It is a recording of the electrical signals that travel through the muscles of the stomach and control the muscle's contraction [12, 13]. EGG used when there is a suspicion that the 
muscles of the stomach or the nerves controlling the muscles are not working normally. EGG done by placing the electrode cutaneously over the stomach and the electrical signals coming from the stomach's muscles are sensed by the electrode and recorded on a computer for analysis by lying patient quietly. In normal individuals the EGG is a regular electrical rhythm generated by the muscles of the stomach and the power (voltage) of the electrical current increases after the meal. In patients with abnormalities of the muscles or nerves of the stomach, the rhythm often is irregular or there is no post-meal increase in electric power. EGG will not have any side effects and it is painless study. The EGG wave with and without noise is shown in fig. 1 .

\section{The Anatomy of the stomach}

The main function of the stomach is to process and transport food $[2,6]$. The contractile activity of the stomach takes place when the food enter and it helps to mix, grind and eventually evacuate small portions of chyme into the small bowel, while the rest of the chyme is mixed and ground. Anatomically, the stomach can be divided into three major regions: fundus (the most proximal), corpus and antrum. The density of the smooth muscle cells increases in the antral area. The area in the corpus around the greater curvature, where the split of the longitudinal layers takes place is considered to be anatomically correlated with the origin of gastric electrical activity. The stomach wall is like a wall of most other parts of the digestive canal, consists of three layers: the mucosal (the innermost), the muscular is and the serosal (the outermost). The mucosal layer it can be divided into three layers: the mucosa (the epithelial lining of the gastric cavity), the muscularis mucosae (low density smooth muscle cells) and the submucosal layer (consisting of connective tissue interlaced with plexi of the enteric nervous system). The second gastric layer, the muscularis, can also be divided into three layers: the longitudinal (the most superficial), the circular and the oblique. The longitudinal layer of the muscularis can be separated into two different categories: a longitudinal layer that is common with the esophagus and ends in the corpus, and a longitudinal layer that originates in the corpus and spreads into the duodenum as shown in Fig 1.

\section{Electrodes Positioning}

The electrical signals are generally produced in the mid-corpus of the stomach where the electrical activity takes place. Fig. 3 (A) and Fig.3 (B) represents the positioning of the $\mathrm{Ag} / \mathrm{Agcl}$ electrodes for tapping of these signals. Two electrodes $A$ and $B$ are placed in the fundus and the mid corpus (Body) of the stomach. The third electrode $C$ is placed as ground at the end of the stomach region (Antrum) for patient safety [3, 4, 5].

\section{Materials and Methods \\ EGG data is recorded with the} electrogastrography setup among the outpatient's gastroenterology clinic and the student with disorders were studied for this investigation at biomedical department of our institution and gastroenterology department of a reputed hospital. More than hundred numbers, includes patients, normal subjects in both male and female category of different age groups are participated in this recording $[8,10]$. The patients included 40 with Dyspepsia, 45 with Stomach ulcer, and 30 with Nausea, 35 with Bradygastria and 30 with tachygastria are included for this analysis. This acquisition of data started before four years and till date to for strong database. The various categories of the subjects are listed in Table 1.

The electrodes are positioned as described in the section 4 of this paper to record the data. The data are acquired via data scope and the same is stored as data base in the computer for further analysis. In this paper, EGG data are reconstructed as an original EGG signal and the same is included in the principle component analysis [PCA] to get actual EGG signal. Denoising of the half an hour recorded EGG signal a subject with digestive disorder is performed with wavelet denoising technique as shown in fig. 4 to find the frequency and power spectrum.

A recoding setup is proposed to acquire the electrical signals cutaneously from the stomach. The electrodes are well protected within a shield to reduce electromagnetic interference. EGG from electrode is undergoes for signal conditioning in SCU unit, which include instrumentation amplifier (IA) and filter. IA is high input impedance and high CMRR about $100 \mathrm{~dB}$ with variable gain 1 to 10000 . The noise due to respiratory, cardiac and other bio signals along with motion artifacts is also acquired with EGG. This is eliminated in the filter section and the frequency range from 1 to $10 \mathrm{cpm}$ of $E G G$ is viewed in the digital storage scope (DSO). The EGG given to data scope to convert the analog form into digital form to store as database in the $P C$ via RS 232 serial interface for analysis and classification to assist the physician in the investigation of digestive system disorder. The general block diagram for the above said proposed recoding setup is shown in fig. 5

\section{Acquisition procedure for EGG}

1. Lightly abrade the skin with abrasive pads, and put a spot of gel on the electrode contact area.

2. Two electrodes are placed on the Fundus and the mid-corpus and a reference 
electrode is placed on the right side of the abdomen.

3. The output of the electrodes is given to the Instrumentation amplifier of the EGG recording unit after the setup is calibrated.

4. The amplified output signal is given to the filtering section of the recording setup.

5 . The output of the filter is recorded as an EGG data via a data scope to form a database of digestive disorder patients.

6. The EGG data from the database is reconstructed as an original EGG signal. This signal undergoes PCA analysis before denoising. 7. These data are displayed as signal after wavelet transform on the PC using MATLAB program.

8. Welch power spectral Density Estimation is adopted to find the frequency in $\mathrm{Hz}$ and the power in $\mathrm{db}$ of the denoised signal.

9. The threshold for detection of various disorders was fixed based on the observations and in consultation with the physician as 0.06 $\mathrm{Hz}$ to $0.075 \mathrm{~Hz}$ for ulcer patient $0.02 \mathrm{~Hz}$ to 0.04 $\mathrm{Hz}$ for dyspepsia patient and $0.05 \mathrm{~Hz}$ to $0.058 \mathrm{~Hz}$ for Normal Individual.

\section{Results and Discussion}

The EGG data acquired for the different subjects as discussed in the section Materials and Method is considered for analysis. The denoising of the EGG signal using Wavelet transform increases the reduction of noise signal which is below or above EGG frequency limit (3cpm or $0.05 \mathrm{~Hz}$ ). From the database, the data are analyzed for detecting frequency and power of the denoised EGG signal of the normal subjects and the subjects with digestive system disorders using Welch power spectral Density Estimation [14]. The fig. 6 shows the range of frequencies and power for the normal individual with frequency $0.047 \mathrm{~Hz}$ and power $47.13 \mathrm{~dB}$, the fig.7 shows the range of frequencies and power for the dyspepsia patient with frequency $0.023 \mathrm{~Hz}$ and power $48.42 \mathrm{~dB}$ and the fig. 8 shows the range of frequencies and power for ulcer patient in ' $C$ ' with frequency $0.061 \mathrm{~Hz}$ and power $44.57 \mathrm{~dB}$. In this study, we have used the EGG setup as described in the section 5 to record myoelectrical activity for the patients suffering from Dyspepsia, Stomach ulcer and normal individual. The frequency and power for the EGG signals are obtained with the help of MATLAB software and the same is compared between normal individual and stomach disorder patients [7, 9]. It is observed from bar graph result if the frequency gets increased the power get decreased for the disorder patients. Power comparison is shown in fig. 9 and the frequency comparison is shown in fig. 10 .

\section{Conclusion and Discussion}

In this paper, we have succeeded in acquiring the EGG from normal individual and abnormal patients who are suffering from different stomach disorders under the monitor and guidance of the gastroenterologist from the reputed hospital and formed a database for EGG signals. The signal analysis is performed using MATLAB software in reconstructing the original $E G G$ signal from the database. The denoising of the same is performed after denoising with help of wavelet transform. The Welch power spectral Density Estimation method is adapted to the denoised EGG signal to find the power and frequency. The power and frequency of the EGG are compared and it is observed that when the power of the signal increased corresponding frequency is decreased for digestive disorder patient when compare to normal individual. This fact may very much support the previous research work done in the field. The future work in this project would be to acquire EGG from patients suffering from different diseases to form a strong database. The signal analysis can be extended further as challenge to the researcher to find best spectral analysis techniques which should detect a minute changes in the frequency in the spectral analysis to a greater extent to study the intricate behaviour of the EGG signals which would help diagnose the diseases more efficiently and precisely. A database for each disease is desired to be created so that sufficient data will be available to diagnose a disease. The future work is done by replacing the $\mathrm{Ag} / \mathrm{Agcl}$ electrode by active electrode to improve the acquisition of EGG signal cutaneously.

\section{Acknowledgement}

The authors acknowledge their indebtedness to the following medical experts $\mathrm{Dr} L$ Venkatakrishnan (M.D., D.M., D.N.B.), Head of Gastroenterology Dept., Dr.J.Krishnaveni (M.D., D.N.B.), Gastroenterologist from PSG Hospitals, Coimbatore, Dr.T.S.Chandrasekar (M.D., D.M.), Interventional Gastroenterologist for their support and for permitting us to use the facilities at the hospitals for live testing of the recording setup and sharing valuable patient database with us. We are also acknowledging the Management of Sri Ramakrishna Engineering College, Coimbatore for their support in acquiring data from the student at the department of biomedical Engineering.

\section{References}

[1] Alvarez W.C. (1922) JAMA, 78, 1116-1118.

[2] Smout A.J.P.M., Van Der Schee E.J., Grashuis J.L. (1980) Digestive Diseases and Sciences 78,179-187.

[3] Abell T.L., Malagelada J.R. (1988) Digestive Diseases and Sciences, .33,982-992. 
[4] Chen J., McCallum R.W. (1991) Medical \&Biological Engineering \& Computing, 29,339-350.

[5] Riezzo G., Pezzolla F., Thouvenot J. (1992) Pathology Biology, 40,889-894.

[6] Mintchev M.P., Kingma Y.J., Bowes K.L. (1993) Gastroenterology, 104, 12731280.

[7] Chen J., McCallum R.W. (1992) American Journal of Gastroenterology, 87,477482.

[8] Pfaffenban B., Adamek R.J., kuhn K. (1995) Digestive Diseases and Sciences, 40,445-450.

[9] Chen J.D.Z., Lin Z., Pan I. (1996) Digestive Diseases and Sciences, 41, 1538-1545.

[10] Chen J.D.Z. (1998) Annual International conference of the IEEE Engineering in Medicine and Biology Society, 20(6),2802-2807

[11] Leahy A., Besherdas K., Clayman C., Mason I., Epstein O. (1999) American Jourl of Gastroenterology, 94, (4), 1023-1028.

[12] Chen D.Z., Zhiyue Lin, Ed. (2006) Electrogastrogram-Encyclopedia of Medical Devices and Instrumentation.edited by John G. Webster.United Kingdom: John Wiley \& Sons, Inc.2006.

[13] Gopu G., Neelaveni R., Porkumaran K. (2008) ICCCE '08 proceeding international conference on Computer and Communication Engineering,13-15 May 2008, Kuala Lumpur, MALAYSIA, 2,201-205.

[14] Gopu G., Neelaveni R., Porkumaran K., Shekar M.G. (2010) Sri Lanka Journal of Bio-Medical Informatics, 1 (1), 20-26. 
Investigation of digestive system disorders with electrogastrogram using wavelet transform denoising

Table 1- Sex and age distribution of patient groups

\begin{tabular}{|l|l|l|l|}
\hline \multicolumn{1}{|c|}{ Disorders } & $\begin{array}{c}\text { Mean } \\
\text { Age } \\
\text { (years) }\end{array}$ & Male & Female \\
\hline Dyspepsia $(\mathrm{n}=40)$ & 38 & 24 & 16 \\
\hline Stomach Ulcer $(\mathrm{n}=45)$ & 34 & 26 & 19 \\
\hline Nausea $(\mathrm{n}=30)$ & 45 & 20 & 10 \\
\hline Bradygastria $(\mathrm{n}=35)$ & 28 & 25 & 10 \\
\hline Tachygastria $(\mathrm{n}=30)$ & 36 & 21 & 9 \\
\hline
\end{tabular}

A

D
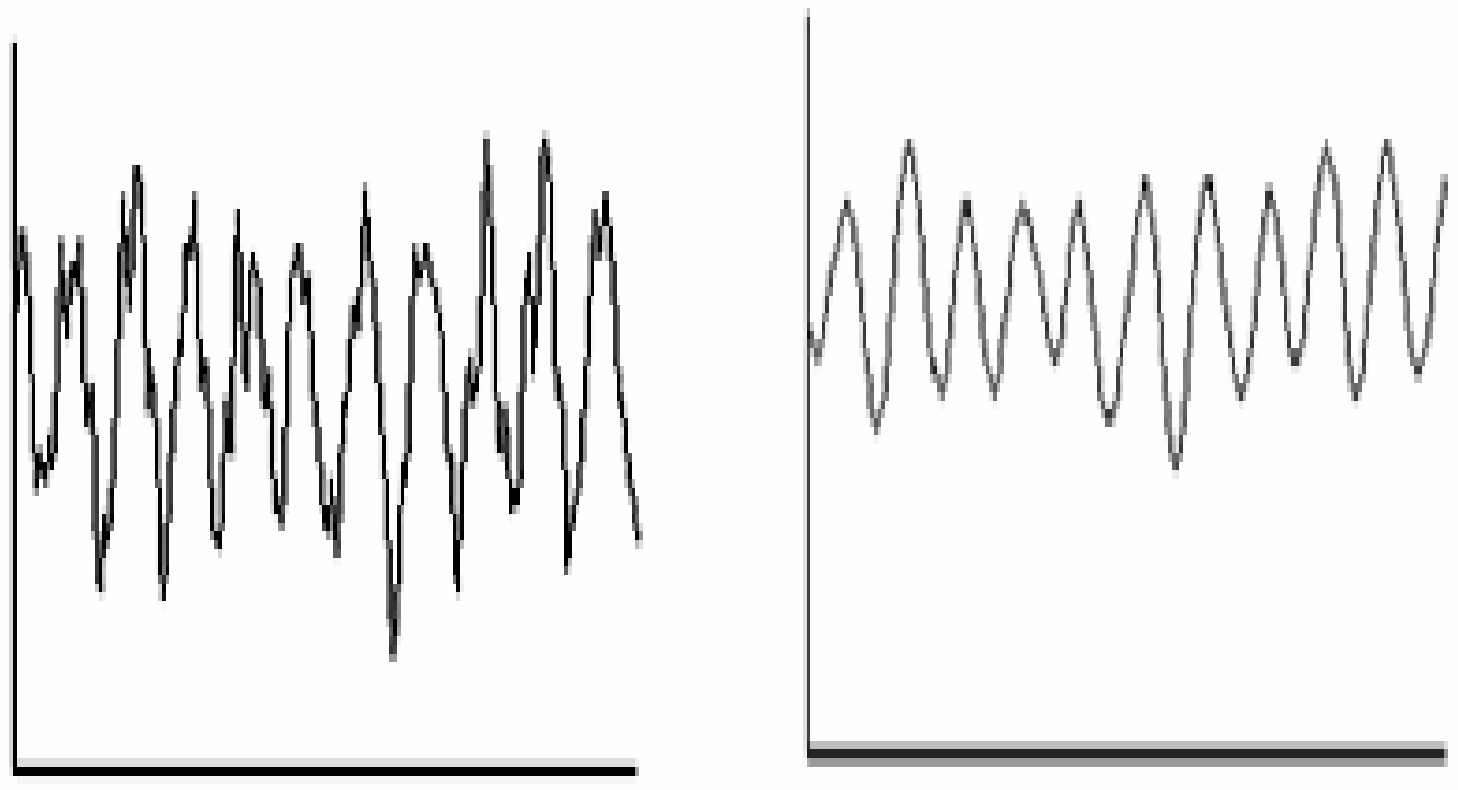

Fig. 1- $A$ and $B$ are EGG waveform with and without noise respectively 


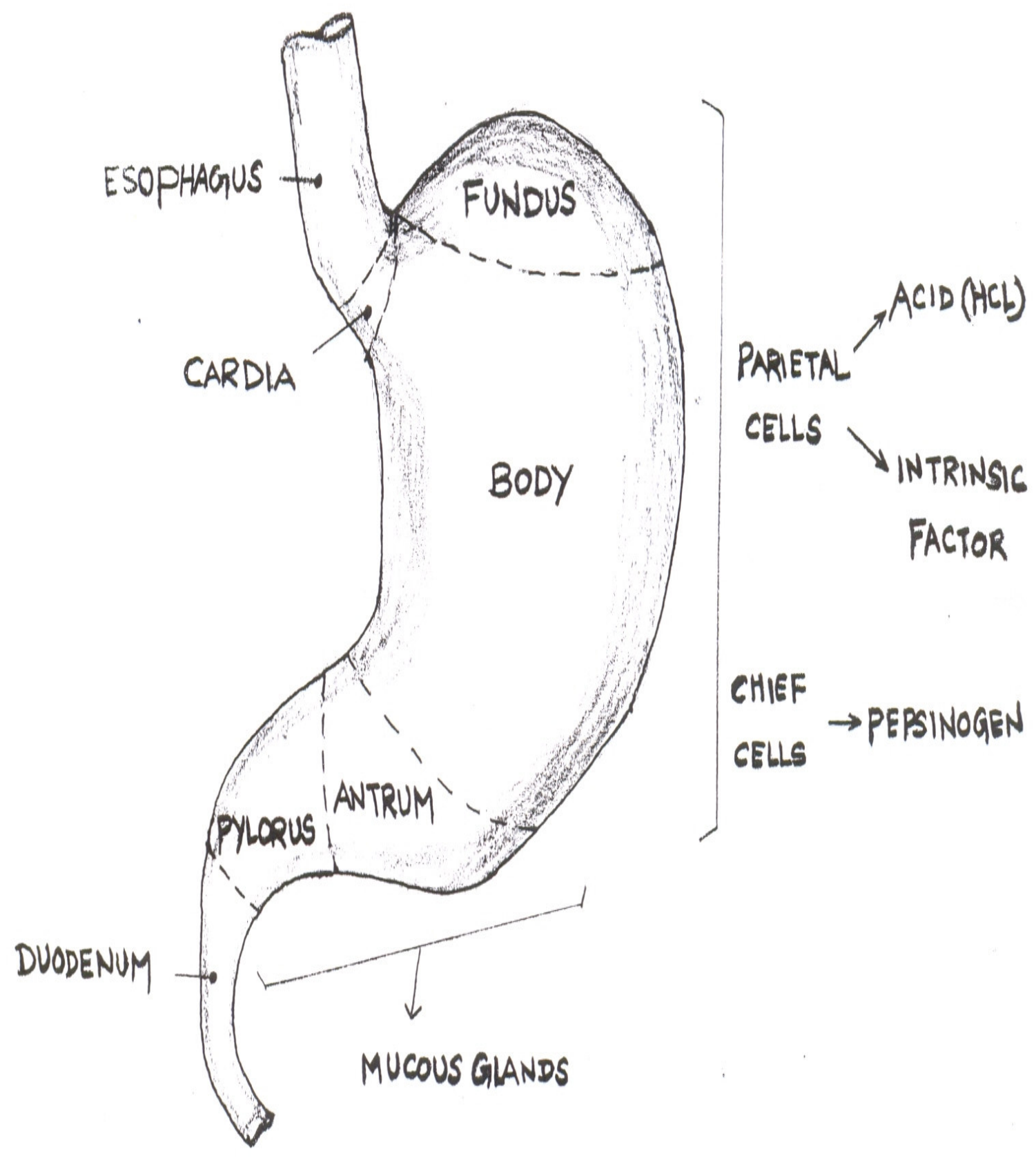

Fig. 2- The Anatomy of the Stomach 

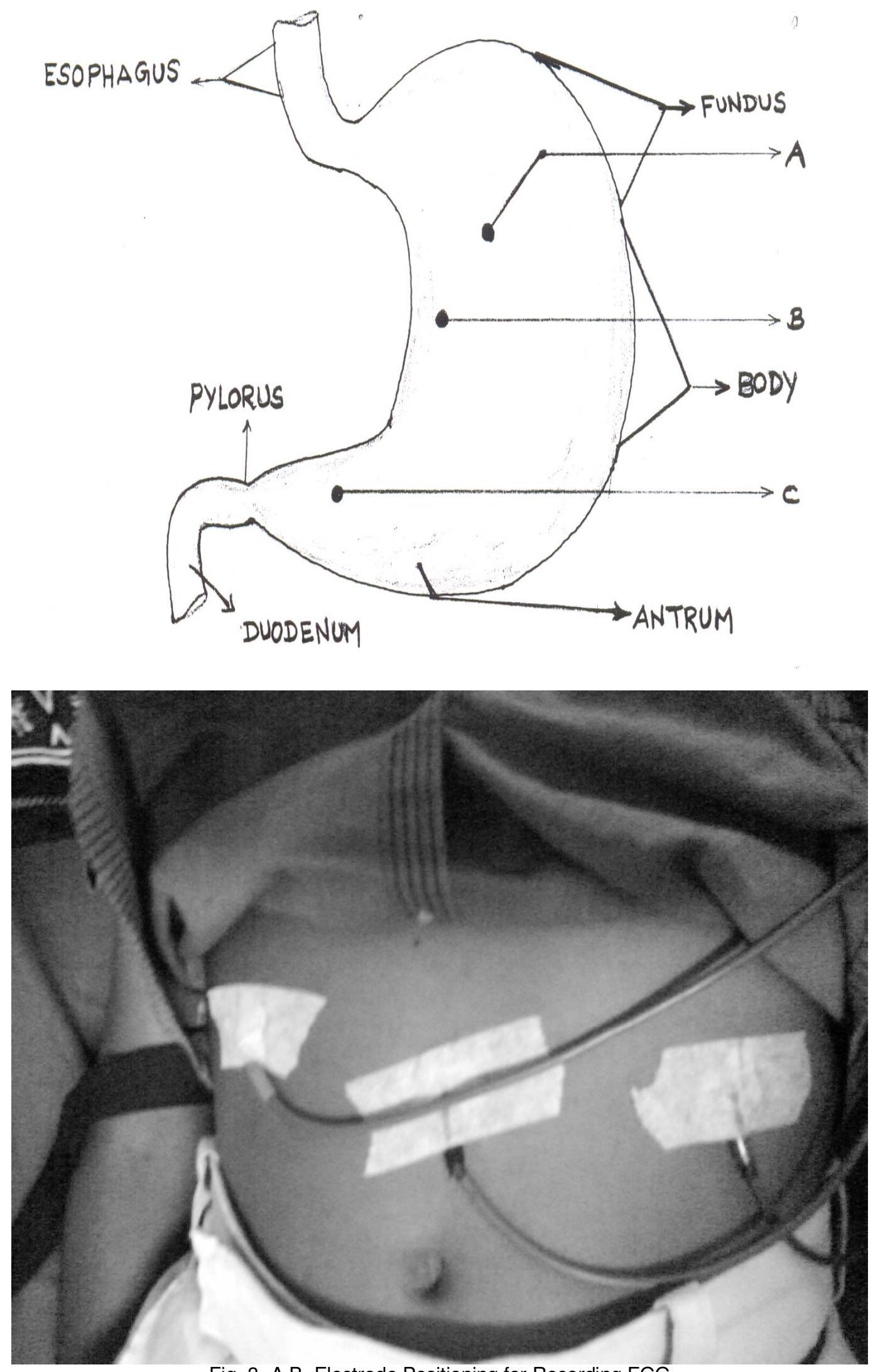

Fig. 3- A,B- Electrode Positioning for Recording EGG 

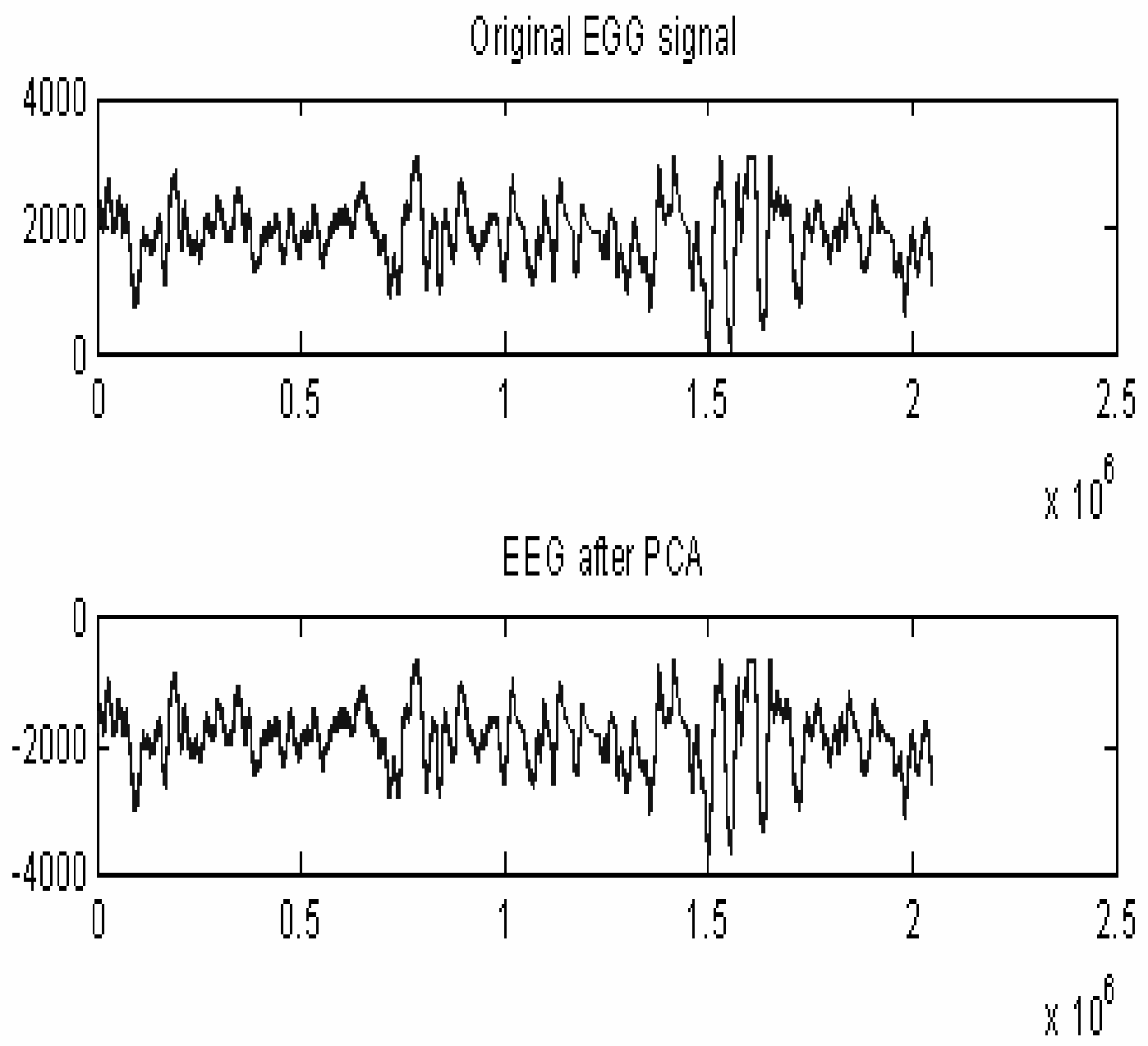

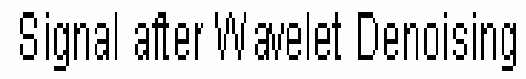

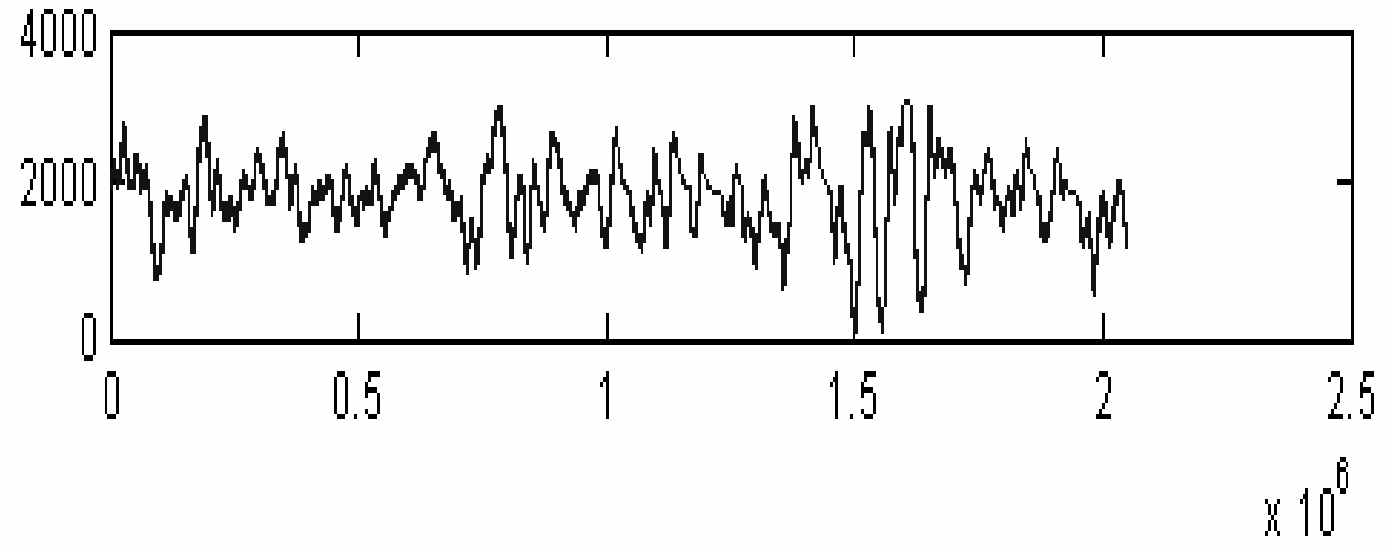

Fig. 4- Denoising of EGG signal with wavelet transforms 
Stomach

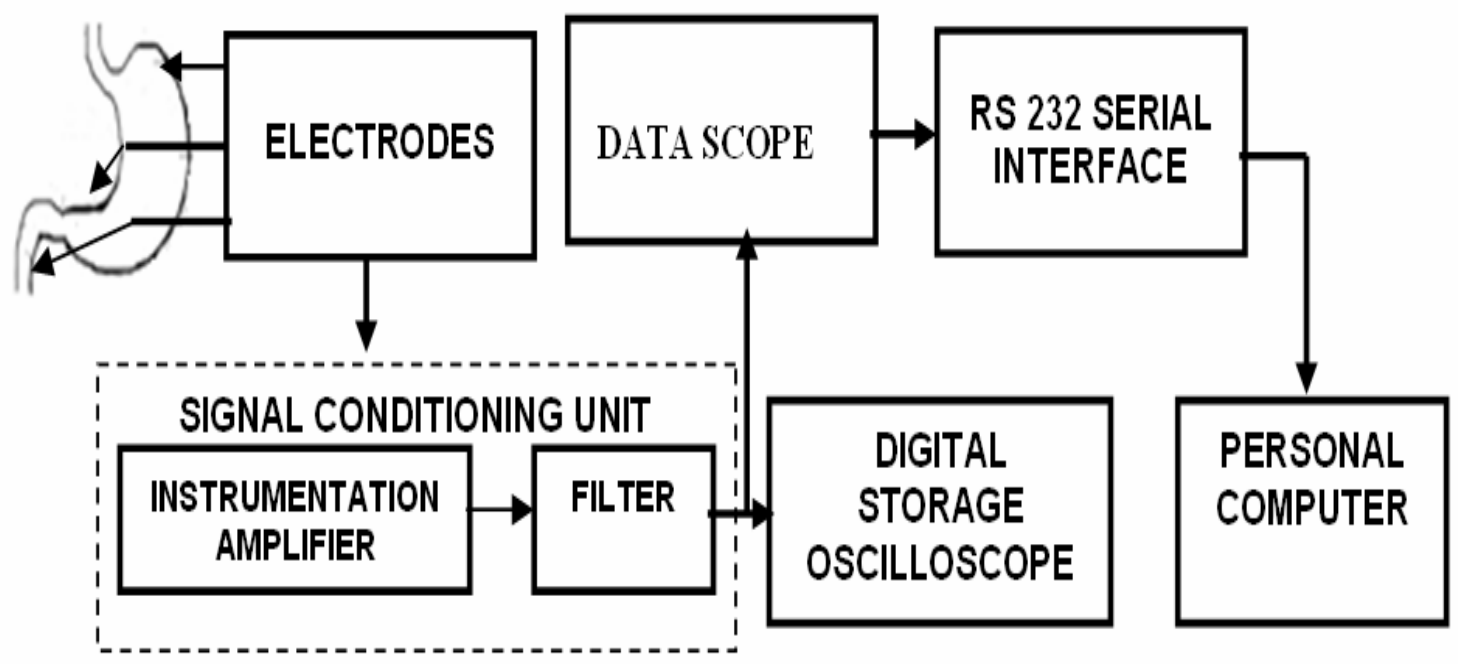

Fig. 5- General Block Diagram

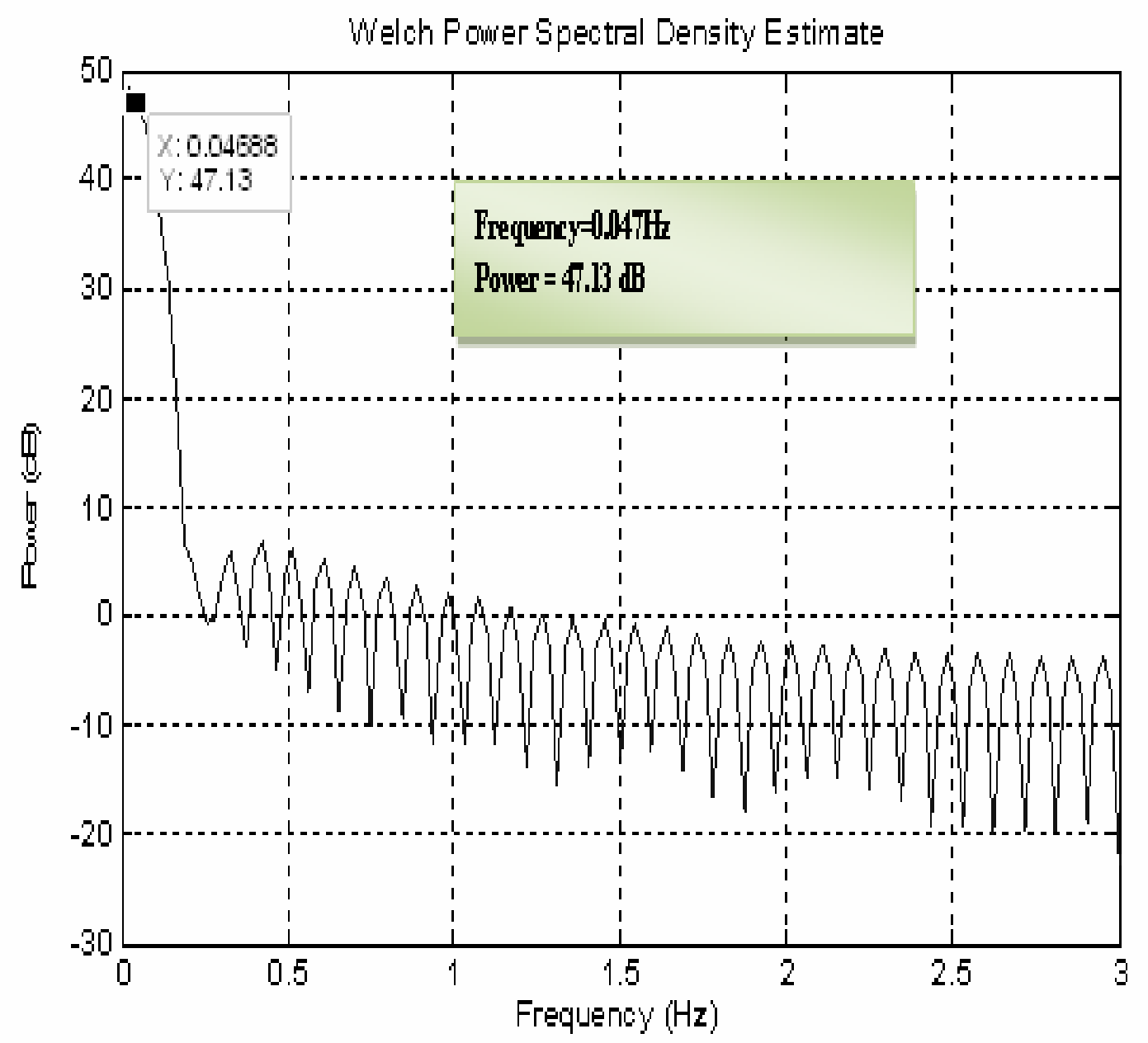

Fig. 6- Welch power spectral Density Estimation for Normal Subject 


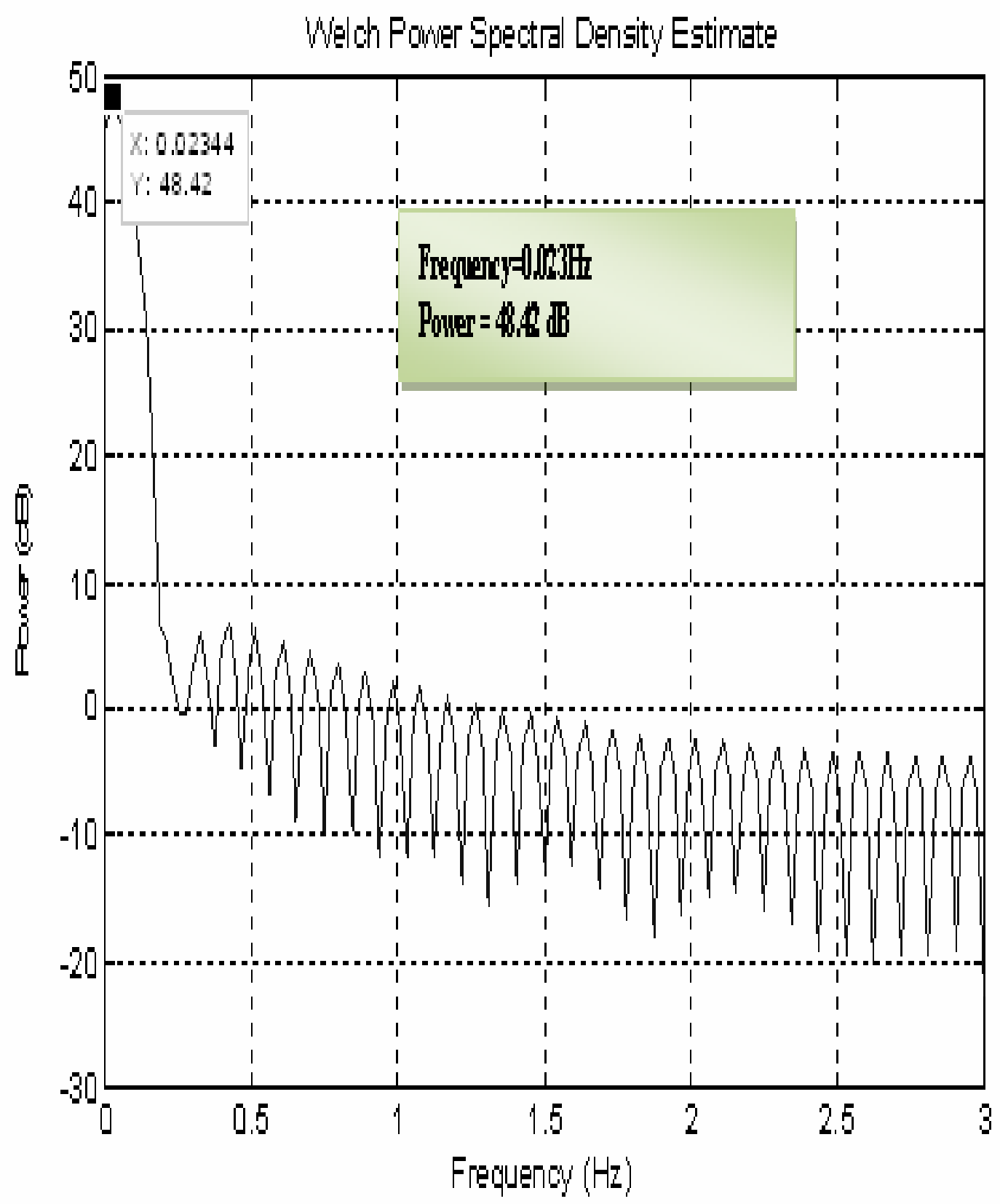

Fig. 7- Welch power spectral Density Estimation for Dyspepsia Patient 


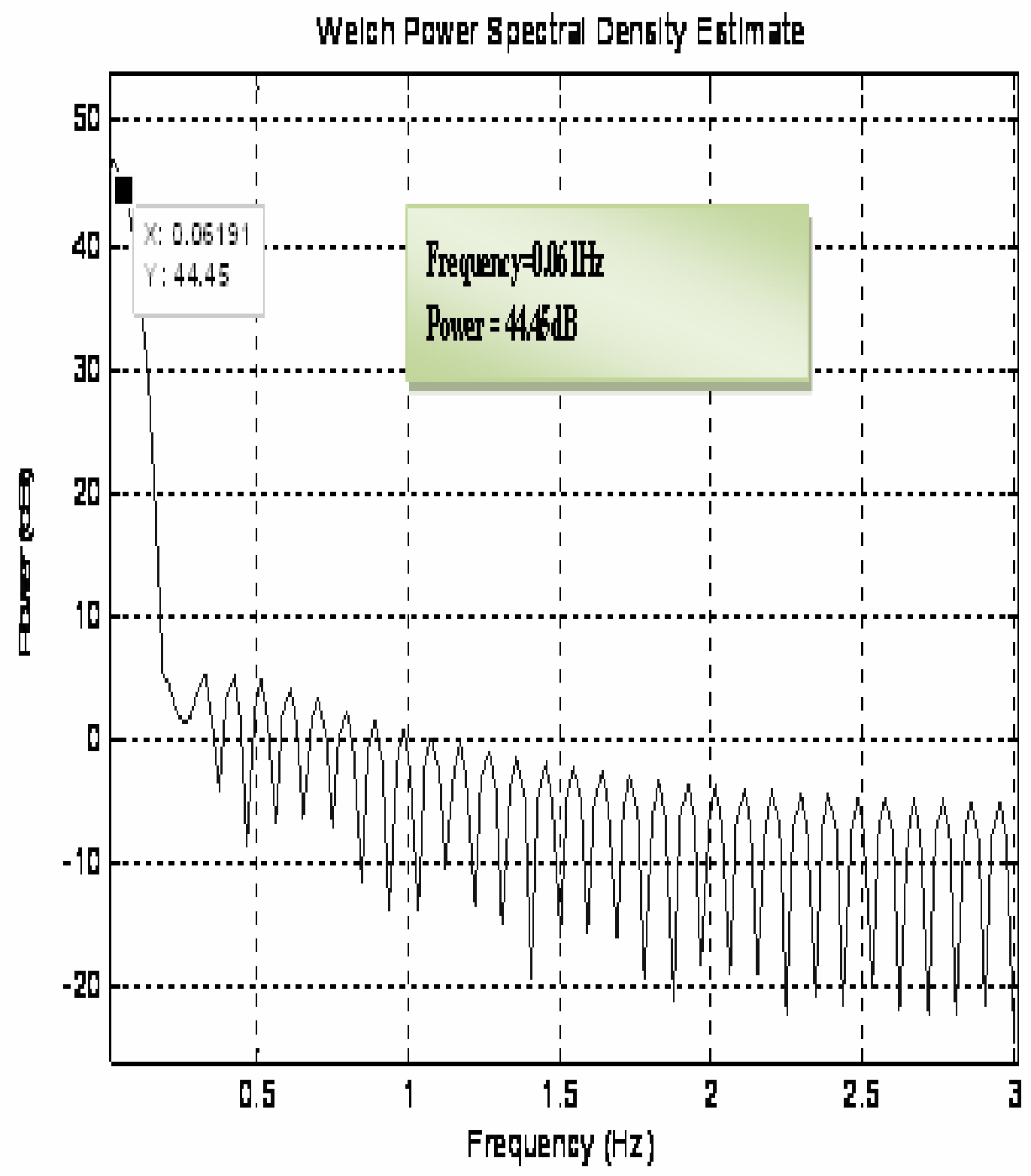

Fig. 8- Welch power spectral Density Estimation for Ulcer Patient 


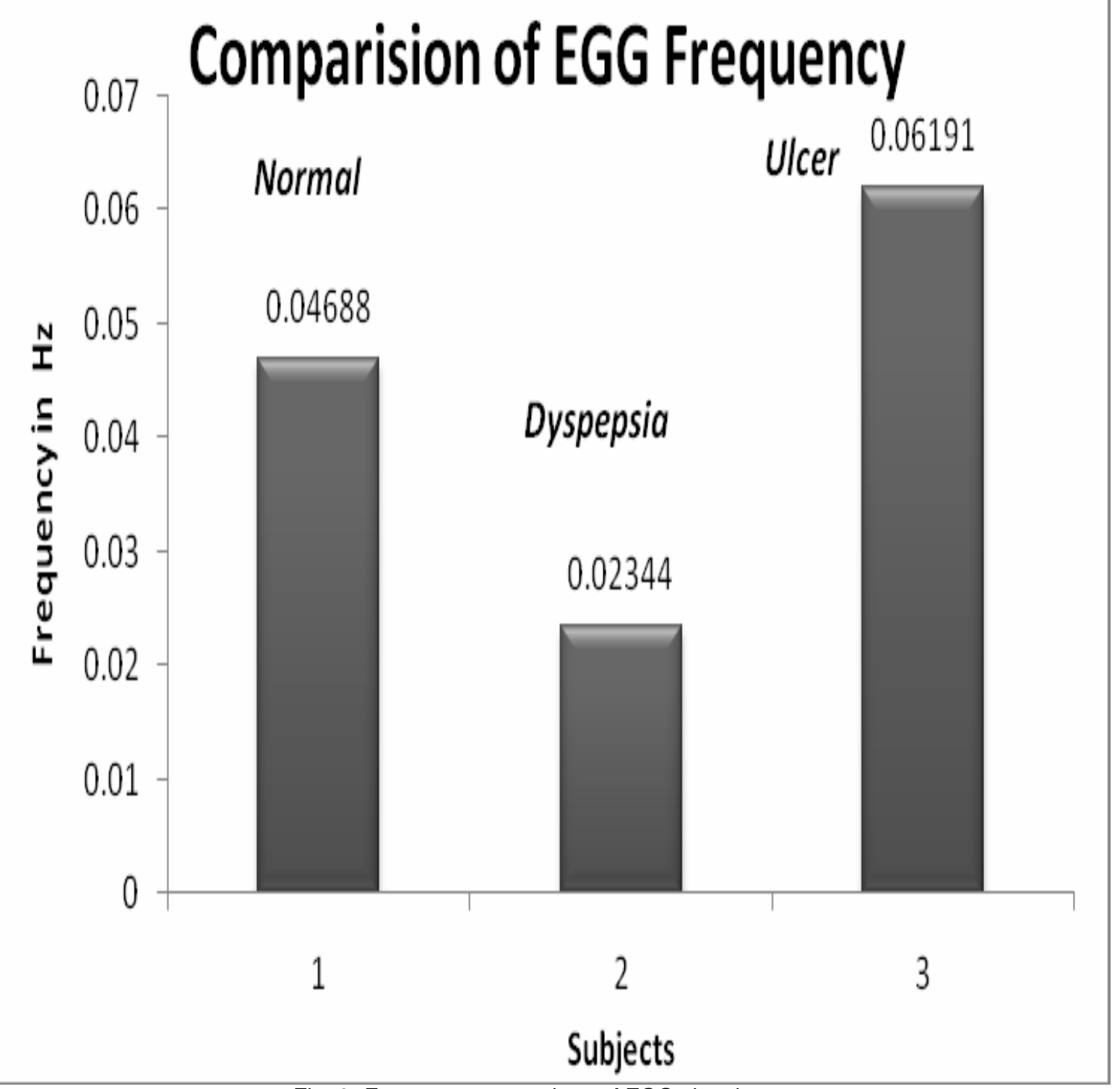

Fig. 9- Frequency comparison of EGG signals 


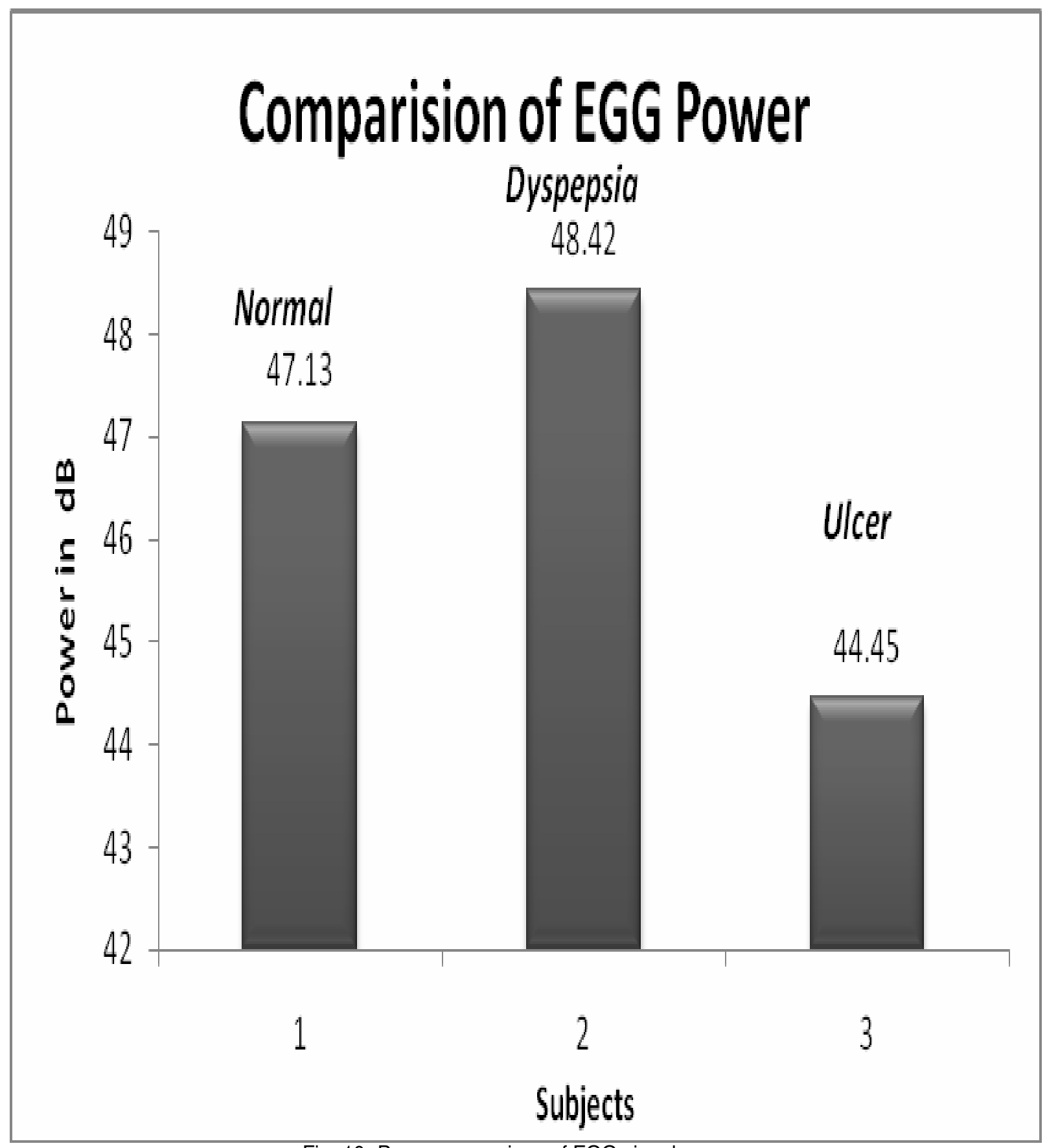

Fig. 10- Power comparison of EGG signals 